\title{
Efficacy and safety of short course adjuvant trastuzumab combination chemotherapy in breast cancer
}

\author{
Sachin S. Hingmire, Mahesh B. Sambhus, Dhananjay S. Kelkar, Sujit Joshi, Purvish M. Parikh', R. Bharath²
}

\begin{abstract}
Background: The adjuvant short course 9-week trastuzumab combination therapy for human epidermal receptor 2 positive breast cancer patients may often be considered as a cost-effective and safe option and has important implications for the Indian subcontinent as well as other developing countries. However, such regimens of shorter duration trastuzumab therapy like FinHer, offered in view of economic constraints, may not be able to achieve globally comparable cure rates in early breast cancer especially with high-risk women with more than 3 lymph node positive. Methods and Material: Outcome of $2 \mathrm{I}$ patients with HER2 positive breast cancer was treated with short course trastuzumab combination chemotherapy in the adjuvant setting was studied. Results: Out of 21 patients 15 are alive and disease free with a follow up of up to 73 months (median follow up 42 months).

Key words: FinHer, short course trastuzumab, survival
\end{abstract}

\section{Introduction}

In 2012, a total of 1.7 million women developed breast cancer globally and a staggering 6.3 million women who had been detected to have this disease in the preceding 5 years were alive. ${ }^{[1]}$ In the USA, the 5 -year overall survival (OS) for breast cancer has increased from $75 \%$ in 1970 s to almost $89 \%$, presently. ${ }^{[2]}$ Unfortunately, this is not replicated in developing countries. For instance, the corresponding figure is about $60 \%$ for India.

The magnitude of the problem shall continue to increase, the Indian Council for Medical Research Cancer Atlas project confirming that, in 2008 , that there was a $26.6 \%$ increase in the total number of cancer cases to be registered from the five cities of Bengaluru, Chennai, Delhi, Bhopal, and Mumbai. ${ }^{[3,4]}$

The hospital-based registry data of 2008 from the largest cancer center in India, reported on 2001 new patients of breast cancers. Estrogen receptor (ER) and/or progesterone receptor (PR) expression was positive in tumors of 1025 (51.2\%) patients. Human epidermal receptor 2 (HER2) $3+$ expression by immunohistochemistry (IHC) was found in $335(16.7 \%)$ and HER2 $2+$ in $163(8.1 \%)$. The triple negative phenotype was found in $596(29.8 \%)$ patients. An estimated 441 patients were eligible to receive HER2-targeted therapy based on their HER2 status. Of these $38(8.6 \%)$ patients received some form of HER2-targeted therapy; twenty patients $(4.5 \%)$ as part of ongoing clinical trials and $18(4.1 \%)$ as part of routine care. ${ }^{[5]}$

The cost of therapy is, therefore, a major limiting factor. To improve the outcome of breast cancer in India, we adapted the FinHer schedule in HER2 positive breast cancer patients who had successfully undergone potentially curative R0 resection and were unwilling to accept the standard 1 year trastuzumab therapy. They were followed up prospectively, and the analysis of this data is being presented here.

\section{Materials and Methods}

Patients with HER2 positive breast cancer who had undergone potentially curative surgery and were given adjuvant short

\begin{tabular}{|l|}
\hline Access this article online \\
\hline Quick Response Code: \\
\\
Website: www.sajc.org \\
\hline DOI: 10.4103 /sajc.sajc_68_17 \\
\hline
\end{tabular}

Department of Oncology, Deenanath Mangeshkar Hospital and Research Centre, Pune,

'Department of Oncology, Asian Institute of Oncology, Mumbai, Maharashtra, ${ }^{2}$ Department of Oncology Kovai Medical Centre and Hospital, Coimbatore, Tamil Nadu, India

Correspondence to: Dr. Sachin S. Hingmire, E-mail: sshingmire@yahoo.com course trastuzumab-chemotherapy combination were followed up prospectively. Detailed history, examination, operative details, and histopathological reports were recorded. HER2 expression was determined by IHC and/or fluorescence in situ hybridization (FISH) and interpreted using standard recommendations. ${ }^{[5,7]}$

Part one of the systemic therapy consisted of three cycles of docetaxel $\left(80-100 \mathrm{mg} / \mathrm{m}^{2}\right.$ as a $1 \mathrm{~h}$ intravenous infusion on day 1 of each 21-day cycle). Simultaneously, nine trastuzumab infusions were administered at weekly intervals, the first infusion being given on day 1 before the first docetaxel. ${ }^{[6]}$ The first dose of trastuzumab $(4 \mathrm{mg} / \mathrm{kg})$ was administered over $90 \mathrm{~min}$, and the subsequent doses ( $2 \mathrm{mg} / \mathrm{kg}$ ) were administered over 30-60 min infusions. Part two of the systemic therapy included three cycles of intravenous (fluorouracil $500 \mathrm{mg} / \mathrm{m}^{2}$, epirubicin $90 \mathrm{mg} / \mathrm{m}^{2}$, and cyclophosphamide $500 \mathrm{mg} / \mathrm{m}^{2}$ [FEC]) also administered on day 1 of each 21-day cycle. No trastuzumab was given during FEC administration or after chemotherapy. Additional supportive care was given as necessary for each patient (antiemetics, growth factors, etc.)

Regular monitoring and blood tests were carried out as per published guidelines and doses reduced, omitted, or postponed as required. Once the systemic chemotherapy was concluded, patients were given appropriate radiation therapy and endocrine therapy if applicable.

Patients were followed up at regular intervals. In case of clinical suspicion of recurrence of breast cancer, appropriate investigative studies were carried out to document the same. Patients were given additional cancer-directed therapy on a case to case basis as required. Follow-up was continued in all patients and status recorded as on April 5, 2016.

\section{Results}

A total of 21 patients with HER2 positive breast cancer who received adjuvant short course trastuzumab combination chemotherapy were evaluated from July 2009 to April

This is an open access article distributed under the terms of the Creative Commons Attribution-NonCommercial-ShareAlike 3.0 License, which allows others to remix, tweak, and build upon the work non-commercially, as long as the author is credited and the new creations are licensed under the identical terms.

For reprints contact: reprints@ @medknow.com

How to cite this article: Hingmire SS, Sambhus MB, Kelkar DS, Joshi S, Parikh PM, Bharath R. Efficacy and safety of short course adjuvant trastuzumab combination chemotherapy in breast cancer. South Asian J Cancer 2017;6:47-50. 
2016. Their age ranged from 40 to 72 years with a median of 62 years. This included 4 premenopausal women and 17 postmenopausal ladies. Four of them had undergone breast conservative surgery, and the remaining 17 had opted for modified radical mastectomy. The surgery was done by an oncosurgeon in 17 of the 21 cases.

Histology confirmed invasive ductal carcinoma in all the cases. It was reported as Grade 1 in one patient, Grade 2 in 11 and Grade 3 in the remaining nine patients. The size of the primary tumor was $\leq 2 \mathrm{~cm}$ tumor in three patients, $2-5 \mathrm{~cm}$ in 17 cases and $>5 \mathrm{~cm}$ in one patient. Of the total 21 patients, axillary lymph node (LN) involvement was seen in 16 (76\%), more than $3 \mathrm{LN}$ were positive in 14 patients. Lymphovascular emboli positivity in 11 and perineural invasion in 5. Three patients had a significant component of ductal carcinoma in situ.

HER2 was $3+$ on IHC in 19 patients and $2+$ in two patients confirmed as positive on FISH. Of these 7 were expressing ER ( 6 of them also being PR positive). The majority were fit with 7 having comorbidities (6 HT, 4 DM, and 1 obesity).

Serious Adverse events included febrile neutropenia, ${ }^{[2]}$ port site infection, ${ }^{[1]}$ and hand-foot syndrome (HFS; 1$)$. None of the patients required dose omission or discontinuation. Dose reduction was necessary in one patient (who had developed HFS). None of the patients experienced any cardiotoxicity.

Additional postoperative local treatment in the form of RT was given to 16 patients. All the hormone receptor positive patients received endocrine therapy following the completion of chemotherapy. This included tamoxifenin 4, anastrozole in 2 , and letrozole in 1 patient.

For the group as a whole, the recurrence-free survival (RFS) ranged from 6 to 73 months with median not yet reached. RFS at 3 years was $71.1 \%$.

Similarly, the OS ranged from 9 to 73 months with median still to be reached. OS at 3 years was $89.9 \%$.

At the last follow-up (as of April 5, 2016), 15 patients remained cancer free and well, four patients had died due to recurrent, progressive breast cancer, and the other two were alive with recurrent disease. All six patients who had a recurrence or died with progressive disease were axillary LN positive with more than 3 LNs involved. Recurrence was noted within 8-30 months after the operation. The brain was the first site of metastatic disease in three out of 6 patients. One patient had local recurrence [Table 1].

\section{Discussion}

Breast cancer is a major and escalating problem for developing countries. Data from local registries in India that show that Globocan data are underestimating breast cancer incidence in India. The Indian Council of Medical Research population-based cancer registry of New Delhi shows the incidence to be $29.3 / 100,000$ of the population in 1994-1995. ${ }^{[8]}$ Moreover, the Indian Cancer Society's Maharashtra population-based registry shows it to be 26.8 per 100,000 of the population in $2001 .{ }^{[4]}$ Both these figures are higher than the $<19.5$ incidence per 100,000 population showed in Globocan 2002 data. ${ }^{[9,10]}$

In India, the age-adjusted incidence shows an increasing trend of $1.6 \%$ from Indian Cancer Society's Mumbai cancer registry (metro region) over a 15 years of period (1996-2010). ${ }^{[1,4]}$ Moreover, the projected increase in India's urban population will be to $>550$ million by 2030 . Correspondingly, there will be a doubling of breast cancer burden from a mean annual figure of 1300 (in 2001-2005) to over 2500 (projected annual incidence for the period 2021-2025).

The incidence of HER2 positive cases comprises a significant portion of this tumor burden- making up 25\%-34\% ${ }^{[5,8]}$ Such patients have a poor prognosis, requires expensive HER2 directed therapy saving lives. ${ }^{[7]}$ Unfortunately, its cost makes it applicable to $<5 \%$ of the eligible population. ${ }^{[5]}$ After endocrine therapy, the only other systemic treatment showing substantial benefit is targeting HER2 with trastuzumab. A recent Cochrane meta-analysis of 6 adjuvant and 2 neoadjuvant randomized controlled trials (RCTs-NSABP B-31, NCCTG N9831, BCIRG 006, HERA, FinHer, PACS-04, Buzdar, and NOAH), showed that mortality is reduced by about $33 \%$ and the risk of relapse is diminished by $40 \% \cdot{ }^{[10]}$ Recommended duration of adjuvant trastuzumab therapy has been 1 year. However, affordability and cost-effectiveness of 1 year therapy has been a concern, especially in Indian patients.

The FinHer trial showed that 9 weeks of trastuzumab is more effective than none at all (control), with no difference in cardiotoxicity or brain metastasis. ${ }^{[11,12]}$ Of the 1010 women in FinHer, 232 were HER2 positive, and they were divided into two equal groups (116 each)- one of them receiving short course 9 weeks trastuzumab. At a median follow-up of 62 months, there was no significant difference in distant disease-free survival (DFS) or OS between patients who received trastuzumab $(n=116)$ and those who did not $(n=116)$. At 5 years, trastuzumab-treated patients had a DFS rate of $83.3 \%$ and an OS rate of $91.3 \%$. In patients who received chemotherapy alone, 5-year DFS and OS rates were $73 \%$ and $82.3 \%$, respectively (hazard ratio $[\mathrm{HR}$ ] for $\mathrm{OS}=0.55 ; 95 \%$ confidence interval $[\mathrm{CI}$, $0.27-1.11 ; P=0.094)$. However, DRFS of patients who received docetaxel, trastuzumab, and FEC was superior to that of patients who received docetaxel and FEC (HR0.32; $95 \%$ CI, 0.12-0.89; P. 029). Any recurrence was noted in $9.3 \%$ of patients treated with Docetaxel/FEC/trastuzumab versus $25.9 \%$ of Docetaxel/FEC-treated patients. Possible determinants for success in this regimen were the early administration of trastuzumab and sequencing of taxanes first before anthracyclines. ${ }^{[13]}$

This attenuated duration of trastuzumab, therefore, became a beacon of hope for Indian patients with financial limitations. ${ }^{[12]}$ In a country, where $<10 \%$ of the population used to have a meaningful medical insurance and $<5 \%$ of eligible patients actually receive trastuzumab, the combination of 9 weekly trastuzumab concomitant with docetaxel followed by FEC may be affordable and effective option with minimal toxicity.

Icli et al. conducted a retrospective analysis comparing DFS in patients who received adjuvant herceptin for 9 weeks versus $>9$ weeks. Investigators reviewed Turkish Ministry of Health records of 690HER2-positive breast cancer patients. Median follow-up was 22 months. A greater percentage of patients in the $>9$-week cohort received either breast-conserving 
surgery $(P=0.015)$ or adjuvant radiotherapy $(P=0.001)$ compared with those in the 9 -week cohort. A higher proportion of node-negative patients $(P=0.002)$ were observed in the 9 -week cohort. At 2 years, DFS in patients who received herceptin for 9 weeks $(n=204)$ was $85.6 \%$ compared with $85.4 \%$ of patients who received herceptin for $>9$ weeks $(n=486$; unadjusted HR $0.934 ; 95 \%$ CI, $0.552-1.580, P=0.28)$. Cardiac toxicity leading to interruption of herceptin therapy was reported in $0 \%$ of patients in the 9 -week group and $3.3 \%$ of patients in the $>9$-week group. ${ }^{[14]}$

In the second retrospective analysis, Tonyali et al. compared results for 479 consecutive women who received adjuvant herceptin for 9 weeks $(n=181)$ or 52 weeks $(n=298)$ at 8 centers. The median duration of follow-up was 30 months. At baseline, the 52-week group had more high-risk patients who had received intensive adjuvant treatment than the 9-week group. Results at 36 months for DFS and OS were similar in the 2 groups. For 9 weeks versus 52 weeks, respectively, the DFS rate was $90 \%$ versus $85 \%(P=0.132)$ and the OS rate was $96 \%$ versus $97 \%(P=0.779)$. Recurrences were observed in $8.3 \%$ versus $11.7 \%$ and death occurred in $2.7 \%$ and $2 \%$ of patients treated for 9 weeks versus 52 weeks, respectively. ${ }^{[15]}$

Sendur et al. retrospectively analyzed patients with HER2-positive, node-positive breast cancer who received adjuvant herceptin for 9 weeks $(n=155)$ and 52 weeks $(n=116) .16$ Median follow-up was $34(4-95)$ months, there was no difference in DFS $(P=0.76)$ and OS $(P=0.99)$ at year 1,3 and $5 .{ }^{[16]}$

Reports of survival rates Indian patients with HER2 positive breast cancer with or without adjuvant trastuzumab therapy are sparse.

In a study, reporting outcome of breast cancer patients from Eastern India, 5 years survival of HER 2 positive patients was only $36.8 \%$. Adjuvant therapy in these patients did not incorporate trastuzumab (2011). ${ }^{[17]}$

DFS of $46.1 \%$ at median follow-up of 40 months was noted in HER2 positive patients treated at a center in Bengaluru, India (2010). ${ }^{[18]}$

Our retrospective study of small number of Indian patients reveals the experience of adjuvant short course trastuzumab/ docetaxel/FEC combination (FinHer like) therapy in breast cancer. Six out of 21 patients developed recurrence after trastuzumab-based adjuvant therapy. All these patients who developed recurrence in spite of incorporation of trastuzumab in the adjuvant regimen however, had higher risk features like more than 3 axillary LN positive. This recurrence rate seems to be higher than expected as per the efficacy rates of adjuvant trastuzumab reported in various global trials, however, may be better than those reported for Indian patients with HER2-positive breast cancer without anti-HER2 therapy.

Trials evaluating shorter duration of trastuzumab therapy have failed to confirm the efficacy as against standard 1 year therapy. The PHARE trial was a Phase 3, randomized, noninferiority study comparing the efficacy and safety of 6-month trastuzumab to 12-month trastuzumab in patients with HER2-positive early breast cancer $\left(n=1690\right.$ patients in each arm). ${ }^{[19]}$ The study failed to show that 2-year DFS, the primary endpoint, was noninferior for patients who received trastuzumab for
Table 1: Comparison of clinically relevant features with outcome

\begin{tabular}{lccc}
\hline & $\begin{array}{c}\text { Alive } \\
\text { disease } \\
\text { free }\end{array}$ & $\begin{array}{c}\text { Alive with } \\
\text { disease/died } \\
\text { with PD }\end{array}$ & Total \\
\hline$n$ & 15 & 6 & 21 \\
BCT/MRM & $3 / 12$ & $1 / 5$ & $4 / 17$ \\
Oncosurgeon/general surgeon & $14 / 1$ & $3 / 3$ & $17 / 4$ \\
ER-PR positive/negative & $4 / 11$ & $3 / 3$ & $7 / 14$ \\
Comorbidities yes/no & $5 / 10$ & $2 / 4$ & $7 / 14$ \\
LN negative/positive & $5 / 10$ & $0 / 6$ & $5 / 16$ \\
LVE negative/positive & $7 / 8$ & $3 / 3$ & $10 / 11$ \\
PNI negative/positive & $14 / 1$ & $2 / 4$ & $16 / 5$ \\
Toxicity yes/no & $1 / 14$ & $3 / 3$ & $4 / 17$ \\
\hline
\end{tabular}

$\mathrm{PD}=$ Progressive disease, $\mathrm{BCT}=$ Breast conservative surgery, $\mathrm{MRM}=$ Modified radical mastectomy, $\mathrm{ER}=$ Estrogen receptor, $\mathrm{PR}=$ Progesterone receptor, $\mathrm{LN}=\mathrm{Lymph}$ node,

$\mathrm{LVE}=$ Lymphovascular emboli, $\mathrm{PNI}=$ Perineural invasion

6 months compared with 12 months. At a median follow-up of 42.5 months, the HR for 2-year DFS for 6 months vs 12 months of trastuzumab, the primary endpoint, was 1.28 with a $95 \%$ CI of $1.05-1.56(P=0.29)$. The 2 -year DFS rate was $93.8 \%$ in the 12 -month group and $91.1 \%$ in the 6-month group. The HR for DFS in the subgroup of ER-positive $(n=1968$; HR $1.23 ; 95 \%$ CI, $0.92-1.65)$ and ER-negative $(n=1,412$; HR 1.34; 95\%CI, 1.02-1.76) patients also favored 12 months of trastuzumab treatment versus 6 months of treatment.

\section{Conclusion}

Adjuvant trastuzumab saves lives and should be offered to all patients with HER2 positive breast cancer (node-positive and node-negative with a tumor size exceeding $1 \mathrm{~cm}$ ) who are planned for adjuvant chemotherapy. One year remains the standard duration of adjuvant trastuzumab therapy recommended. Regimens of shorter duration trastuzumab like FinHer, offered in view of economic constraints in countries like India may not be able to achieve globally comparable cure rates in early breast cancer especially with higher risk factors such as more than $3 \mathrm{LN}$ positive. The results from SOLD, Short-HER, and PERSEPHONE are eagerly awaited with the expectation that they will confirm the FinHer data for the efficacy of short duration trastuzumab therapy.

Financial support and sponsorship

Nil.

\section{Conflicts of interest}

There are no conflicts of interest.

\section{References}

1. Nagrani RT, Budukh A, Koyande S, Panse NS, Mhatre SS, Badwe R. Rural urban differences in breast cancer in India. Indian J Cancer 2014; $51: 277-81$

2. Goss PE, Strasser-Weippl K, Lee-Bychkovsky BL, Fan L, Li J, Chavarri-Guerra $Y$, et al. Challenges to effective cancer control in China, India, and Russia. Lancet Oncol 2014;15:489-538.

3. Parikh PM, Gupta S, Dawood S, Rugo H, Bhattacharyya GS, Agarwal A, et al. ICON 2013: Practical consensus recommendations for hormone receptor-positive Her2-negative advanced or metastatic breastcancer. Indian J Cancer 2014;51:73-9.

4. Dikshit RP, Yeole BB, Nagrani R, Dhillon P, Badwe R, Bray F. Increase in breast cancer incidence among older women in Mumbai: 30-year trends and predictions to 2025. Cancer Epidemiol 2012;36:e215-20.

5. Ghosh J, Gupta S, Desai S, Shet T, Radhakrishnan S, Suryavanshi P, et al. Estrogen, progesterone and HER2 receptor expression in breast tumors of patients, and their usage of HER2-targeted therapy, in a tertiary care centre in India. Indian J Cancer 2011;48:391-6. 
6. Mates M, Fletcher GG, Freedman OC, Eisen A, Gandhi S, Trudeau ME, et al. Systemic targeted therapy for her2-positive early female breast cancer: A systematic review of the evidence for the 2014 Cancer Care Ontario systemic therapy guideline. Curr Oncol 2015;22 Suppl 1:S114-22.

7. Singh R, Gupta S, Pawar SB, Pawar RS, Gandham SV, Prabhudesai S. Evaluation of ER, PR and HER-2 receptor expression in breast cancer patients presenting to a semi urban cancer centre in Western India. J Cancer Res Ther 2014;10:26-8.

8. National Cancer Registry Programme. Three Years Report of Population Based Cancer Registries 2009-2011. New Delhi: Indian Council of Medical Research; 2013.

9. Parkin DM, Bray F, Ferlay J, Pisani P. Global cancer statistics, 2002. CA Cancer J Clin 2005;55:74-108.

10. Pinto AC, Ades F, de Azambuja E, Piccart-Gebhart M. Trastuzumab for patients with HER2 positive breast cancer: Delivery, duration and combination therapies. Breast 2013;22 Suppl 2:S152-5.

11. Joensuu H, Bono P, Kataja V, Alanko T, Kokko R, Asola R, et al. Fluorouracil, epirubicin, and cyclophosphamide with either docetaxel or vinorelbine, with or without trastuzumab, as adjuvant treatments of breast cancer: Final results of the FinHer trial. J Clin Oncol 2009;27:5685-92

12. Purmonen TT, Pänkäläinen E, Turunen JH, Asseburg C, Martikainen JA. Short-course adjuvant trastuzumab therapy in early stage breast cancer in Finland: Cost-effectiveness and value of information analysis based on the 5-year follow-up results of the FinHer trial. Acta Oncol 2011;50:344-52.

13. Isik M, Dizdar O, Altundag K. Two important determinants may play a role in the success of the FinHer trial. J Clin Oncol 2010;28:e334.

14. Paydas S, Basaran G, Saip P, Dogu GG, Eralp Y, Uslu R. et al. Nine Versus 52 Weeks of Adjuvant Trastuzumab in Early Breast Cancer: An Observational Study of the Turkish Oncology Group. Presented at the American Society of Clinical Oncology 2011 Annual Meeting 2011. ASCO \#629;2011.

15. Tonyali O, Coskun U, Sener N, Inanc M, Akman T, Oksuzoglu B, et al. Nine-week trastuzumab treatment versus 52-week trastuzumab treatment for HER2-positive early-stage breast cancer. J Cancer Res Clin Oncol 2012;138:2145-51.

16. Sendur MA, Aksoy S, Ozdemir NY, Yazici O, Zengin N, Altundag K. et al. The efficacy of adjuvant trastuzumab in HER-2 positive breast cancer with axillary lymph node metastases according to the treatment duration. Curr Med Res Opin 2014;30:2535-42. doi: 10.1185/03007995.2014.965775. Epub 2014 Sep 29.

17. Jana D, Mandal S, Mukhopadhyay M, Mitra D, Mukhopadhyay SK, Sarkar DK. Prognostic significance of HER-2/neu and survival of breast cancer patients attending a specialized breast clinic in Kolkata, Eastern India. Asian Pac J Cancer Prev 2012; 13:3851-5.

18. Vaidyanathan K, Kumar P, Reddy CO, Deshmane V, Somasundaram K, Mukherjee G. ErbB-2 expression and its association with other biological parameters of breast cancer among Indian women. Indian J Cancer 2010;47:8-15.

19. Pivot X, Romieu G, Debled M, Pierga JY, Kerbrat P, Bachelot T, et al. 6 months versus 12 months of adjuvant trastuzumab for patients with HER2-positive early breast cancer (PHARE): A randomised phase 3 trial. Lancet Oncol 2013;14:741-8. 